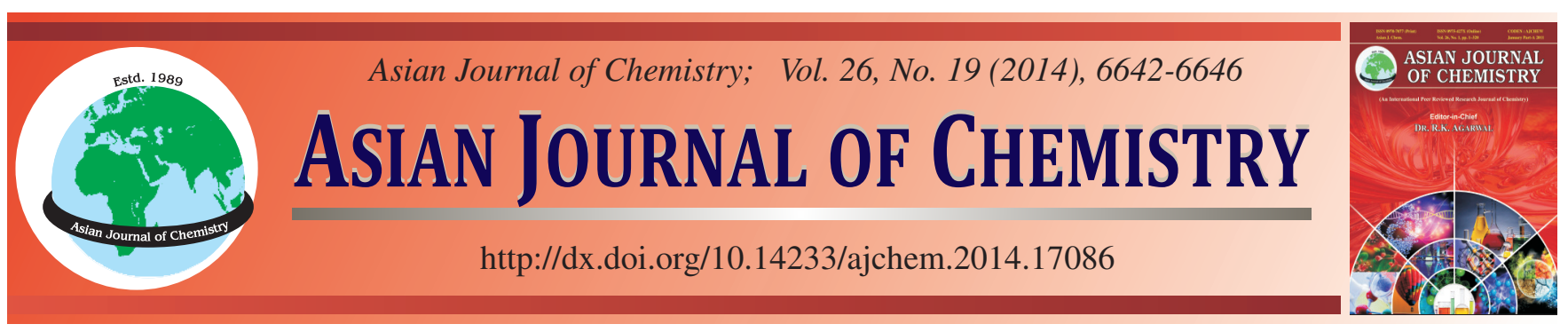

\title{
Synthesis and Antioxidant Activity Studies of Some 5-Chloro-3-substituted 2(3H)-Benzoxazolone Derivatives
}

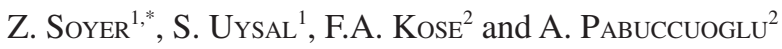

${ }^{1}$ Department of Pharmaceutical Chemistry, Faculty of Pharmacy, Ege University, 35100 Bornova, Izmir, Turkey ${ }^{2}$ Department of Biochemistry, Faculty of Pharmacy, Ege University, 35100 Bornova, Izmir, Turkey

*Corresponding author: Phone: +90 232 3399931; Tel/Fax: +90 23238852 58; E-mail: zeynep.soyer@ ege.edu.tr

2(3H)-Benzoxazolone derivatives are versatile heterocyclic compounds since they display a wide range of pharmacological properties. On the other hand, anilide pharmacophore has been known in medicinal chemistry as an useful template in various pharmacological states. In this study, we synthesized eleven 5-chloro-2(3H)-benzoxazolone-3-alkananilide derivatives and evaluated their antioxidant activities. The structural confirmation of the title compounds was achieved by spectral and analytical data. The antioxidant capacity of the synthesized compounds was determined by the 2,2-diphenyl-2-picrylhydrazyl hydrate (DPPH) and 2,2-azinobis(3-ethylbenzothiazoline6-sulfonic acid) (ABTS) radicals scavenging assays. The results indicated that the majority of the compounds were effective in both tests. The most active compound in the series was compound $\mathbf{6}$, bearing 4-methoxy substituent on the $N$-phenyl ring of the propionanilide skeleton.

Keywords: 2(3H)-Benzoxazolone, Anilide, Antioxidant activity, 2,2-Azinobis(3-ethylbenzothiazoline-6-sulfonic acid), DPPH.

ᄂ - - - - - - - - - - - - - - - - - - - - - - - - - - - -

\section{INTRODUCTION}

Free radicals are generated as a result of normal organ functions or excessive oxidative stress. High level free radicals can cause damage to essential biomolecules (lipids, proteins, enzymes, DNA, etc.) in cells and tissues. The damages by free radicals are involved in the pathogenicity of numerous disorders such as cardiovascular diseases, Alzheimer's and other neurodegenerative diseases, autoimmune diseases, diabetes and cancer $^{1,2}$. Antioxidants are considered as potential drugs because of their ability to delay or prevent the cellular oxidation in these diseases. Minimizing oxidative damage may be an important approach to the prophylaxy or treatment of these diseases, since antioxidants may stop the free radical formation or interrupt an oxidizing chain reaction. This phenomenon has attracted a great deal of interest in research of antioxidant based drugs design. The development of synthetic compounds, capable of scavenging free radicals, has been of great importance, especially in many pathological conditions such as cancer, diabetes, Alzheimer's disease ${ }^{3}$.

Melatonin ( $N$-acetyl-5-methoxytryptamine) (Fig. 1 ), is a pineal hormone that regulates circadian rhythms and also protects macromolecules against oxidative stress which induces tissue damage, lipid peroxidation and apoptosis. The direct and indirect antioxidant effects of melatonin have been well established. Melatonin has been known as a potent free radical scavenger with the ability to remove reactive oxygen species (ROS). Free radical scavenging ability of melatonin has implications for a variety of diseases, including age associated neurodegenerative diseases and cancer initiation. Due to its free radical scavenger and antioxidant properties, melatonin related compounds such as melatonin metabolites and synthetic analogues are under investigation to determine possible leaders which exhibit the highest activity with the lowest side effects ${ }^{4-6}$.<smiles>COc1ccc2[nH]cc(CCNC(C)=O)c2c1</smiles>

Fig. 1. Melatonin

It is well known that small heterocyclic rings act as highly functionalized scaffolds. These are known as pharmacophores of a number of biologically active and medicinally useful molecules ${ }^{7}$. Among these structure, the $2(3 H)$-benzoxazolone heterocycle has high flexibility in chemical modifications, allowing changes to the characteristic of side-chains on rigid platform. On the other hand, the derivetization on nitrogen 
atom of benzoxazolone ring is of interest since the electronic characteristic of corresponding position are known as decisive factor for the biological activity ${ }^{8}$. As a result, this template has broad therapeutic applications. Therefore, the $2(3 H)$-benzoxazolone derivatives exhibit various biological activities such as anti-HIV, anticancer, analgesic, antiinflammatory, antinociceptive, anticonvulsant, antimalarial, human leukocyte MPO chlorinating inhibitor activity ${ }^{9-17}$. Literature survey revealed that the 2(3H)-benzoxazolone heterocycle is endowed with antioxidant properties mediated by radical scavenger ability and 6-methoxy-2(3H)-benzoxazolone was reported as a derivative exhibiting an antioxidative potency similar to that of melatonin $^{18,19} .2(3 H)$-Benzoxazolone scaffold is ring isoster of indol of melatonin. Due to the similarity between the indole of melatonin and benzoxazole rings, it's can be expected that the compounds bearing 2(3H)-benzoxazolone nucleus can exhibit antioxidant activity.

In the light of these findings, in this preliminary study, we designed and synthesized a group of 5-chloro-2(3H)-benzoxazolone derivatives and evaluated their antioxidant properties.

\section{EXPERIMENTAL}

Melting points were determined on an Electrothermal IA 9100 (Electrothermal, Essex, U.K.) melting point apparatus and are uncorrected. The IR spectra of compounds were recorded on a Perkin Elmer FT-IR (ATR) Spectrometer 100 (Perkin Elmer Inc., Massachusetts, USA). The ${ }^{1} \mathrm{H}$ NMR spectra were recorded on a Varian As 400 Mercury Plus NMR (Varian Inc., Palo Alto, CA, USA) spectrometer using DMSO-d6 as solvent. Chemical shifts were reported in parts per million ( $\delta$ ). J values were given in Hz. Mass spectra (ESI-MS) were measured on a Thermo MSQ Plus LC/MS (Thermoscientific Inc., San Jose, CA, USA). Elemental analyses (C, H and N) were performed by Leco TruSpec Micro (Leco, St. Joseph, MI, USA). The analytical results for the elements were within $\pm 0.4 \%$ of the theoretical values.

5-Chloro-2(3H)-benzoxazolone ${ }^{20}$ and $\omega$-chloroanilides ${ }^{21}$ were prepared according to the reported procedures.

General procedure for the synthesis of compounds (1-11): 5-Chloro-2(3H)-benzoxazolone $(0.0012 \mathrm{~mol})$ and $\omega$-chloroanilides $(0.001 \mathrm{~mol})$ were refluxed in DMF in the presence of potassium carbonate $(0.002 \mathrm{~mol})$. By monitoring with TLC, the reaction was terminated. The reaction mixture was poured into cold water. The precipitate was filtered and washed with water. The crude product was crystallized from ethanol:water (1:1) to yield the target compounds.

2-(5-Chloro-2-oxobenzo[d]oxazol-3(2H)-yl)- $\mathrm{N}$-phenylacetamide (1): Yield $40 \%$; reflux time: 46 h; m.p. $215^{\circ} \mathrm{C}$; IR $\left(\mathrm{KBr}, v_{\max }, \mathrm{cm}^{-1}\right)$ (FT/ATR): 3343, 1769, 1698. ${ }^{1} \mathrm{H}$ NMR (DMSO-d $d_{6}$ : $\delta 4.46$ (s, 2H, $\mathrm{CH}_{2}$ ), 4.73 (s, 2H, $\mathrm{CH}_{2}$, min.), 6.956.97 (m, 2H, Ar-H, min), 7.06-7.10 (m, 1H, Ar-H, min.), 7.197.26 (m, 4H, Ar-H, min), 7.30-7.35 (m, 2H, Ar-H), 7.40-7.44 (m, 3H, Ar-H), 7.49-7.58 (m, 3H, Ar-H), 7.49-7.58 (m, 1H, Ar-H, min), 10.37 (brs, 1H, NH) ppm. ESI-MS m/z (\%): 303 $\left([\mathrm{M}+\mathrm{H}]^{+}, 8\right), 305\left([\mathrm{M}+\mathrm{H}+2]^{+}, 3\right)$. Anal. calcd. for $\mathrm{C}_{15} \mathrm{H}_{11} \mathrm{~N}_{2} \mathrm{O}_{3} \mathrm{Cl}$ : C, 59.52; H, 3.66; N, 9.25. Found: C, 59.58; H, 3.83; N, 9.20.
2-(5-Chloro-2-oxobenzo[d]oxazol-3(2H)-yl)- $\mathrm{N}-(4-$ methoxyphenyl)acetamide (2): Yield $97 \%$; reflux time: 89 h; m.p. $224{ }^{\circ} \mathrm{C}$; IR (KBr, $v_{\max }, \mathrm{cm}^{-1}$ ) (FT/ATR): 3325, 1749 , 1680. ${ }^{1} \mathrm{H}$ NMR (DMSO- $\left.d_{6}\right): \delta 3.72\left(\mathrm{~s}, 3 \mathrm{H}, \mathrm{OCH}_{3}\right), 3.65$ (s, $\left.3 \mathrm{H}, \mathrm{OCH}_{3}, \mathrm{~min}\right), 4.44$ (s, 2H, $\left.\mathrm{CH}_{2}\right), 4.70$ (s, 2H, $\left.\mathrm{CH}_{2}, \mathrm{~min}\right)$, 6.89 (d, $2 \mathrm{H}, J=8.8$, phenyl-H, $\min ), 6.95$ (d, $1 \mathrm{H}, J=8.3$, benzoxazolone-H), 7.05 (d, 2H, $J=9.2$, phenyl-H), 7.19 (dd, $1 \mathrm{H}, J=2.4 ; 8.4$, benzoxazolone- $\mathrm{H}, \mathrm{min}), 7.23(\mathrm{dd}, 1 \mathrm{H}, J=$ 2.8; 8.9, benzoxazolone- $\mathrm{H}), 7.32(\mathrm{~d}, 2 \mathrm{H}, J=8.8$, phenyl- $H$ ), 7.41 (d, $2 \mathrm{H}, J=8.4$, phenyl- $H$, min), 7.47-7.49 (m, $1 \mathrm{H}$, benzoxazolone-H, $\min$ ), 7.48 (d, $1 \mathrm{H}, J=2.8$, benzoxazolone-H), 7.51 (d, $1 \mathrm{H}, J=2.4$, benzoxazolone- $H$, min.), 10.22 (brs, $1 \mathrm{H}$, NH) ppm. ESI-MS m/z (\%): $333\left([\mathrm{M}+\mathrm{H}]^{+}, 34\right), 335([\mathrm{M}+$ $\left.2]^{+}, 10\right)$. Anal. calcd. for $\mathrm{C}_{16} \mathrm{H}_{13} \mathrm{~N}_{2} \mathrm{O}_{4} \mathrm{Cl} \cdot 0.3 \mathrm{H}_{2} \mathrm{O}: \mathrm{C}, 56.78 ; \mathrm{H}$, 4.02; N, 8.28. Found: C, 57.01; H, 4.28; N, 7.91.

2-(5-Chloro-2-oxobenzo[d]oxazol-3(2H)-yl)- $\mathrm{N}$-(4chlorophenyl)acetamide (3): Yield $63 \%$; reflux time: $42 \mathrm{~h}$; m.p. $226^{\circ} \mathrm{C}$; $\left(\mathrm{KBr}, v_{\max }, \mathrm{cm}^{-1}\right)$ (FT/ATR): 3348, 1773, 1685. ${ }^{1} \mathrm{H}$ NMR (DMSO- $d_{6}$ ): $\delta 4.46$ (s, 2H, $\mathrm{CH}_{2}, \mathrm{~min}$ ), 4.73 (s, 2H, $\left.\mathrm{CH}_{2}\right), 6.96(\mathrm{~d}, 1 \mathrm{H}, J=8.6$, benzoxazolone- $H, \mathrm{~min}), 7.18-7.26$ (m, 2H, benzoxazolone- $H$, min), 7.37-7.50 (m, 4H, phenyl$\mathrm{H}$, min), 7.37-7.50 (m, 4H, phenyl- $H$ ), 7.53 (d, $1 \mathrm{H}, J=2.4$, benzoxazolone- $H), 7.58-7.61(\mathrm{~m}, 2 \mathrm{H}$, benzoxazolone- $H)$, 10.52 (brs, $1 \mathrm{H}, \mathrm{NH})$ ppm. ESI-MS $m / z(\%): 337\left([\mathrm{M}+\mathrm{H}]^{+}\right.$, 42), $339\left([\mathrm{M}+\mathrm{H}+2]^{+}, 10\right)$. Anal. calcd. for $\mathrm{C}_{15} \mathrm{H}_{10} \mathrm{~N}_{2} \mathrm{O}_{3} \mathrm{Cl}_{2}$ : C, 53.44; H, 2.99; N, 8.31. Found: C, 53.27; H, 2.95; N, 8.48.

2-(5-Chloro-2-oxobenzo[d]oxazol-3(2H)-yl)- $\mathrm{N}$-(4nitrophenyl)acetamide (4): Yield $33 \%$; reflux time: $42 \mathrm{~h}$; m.p. $259^{\circ} \mathrm{C}$; IR (KBr, $\left.v_{\max }, \mathrm{cm}^{-1}\right)$ (FT/ATR): 3341, 1769, 1698. ${ }^{1} \mathrm{H}$ NMR (DMSO- $d_{6}$ ): $\delta 4.49$ (s, 2H, $\mathrm{CH}_{2}, \mathrm{~min}$ ), $4.79\left(\mathrm{~s}, 2 \mathrm{H}, \mathrm{CH}_{2}\right.$ ), $6.96(\mathrm{~d}, 2 \mathrm{H}, J=8.7$, phenyl- $H, \min ), 7.18(\mathrm{dd}, 1 \mathrm{H}, J=1.9 ; 8.3$, benzoxazolone- $H$ ), 7.20-7.24 (m, $2 \mathrm{H}$, benzoxazolone- $\mathrm{H} \mathrm{min}$ ), $7.40(\mathrm{~d}, 1 \mathrm{H}, J=8.3$, benzoxazolone- $H$ ), 7.48 (d, $1 \mathrm{H}, J=2.7$, benzoxazolone- $H, \mathrm{~min}), 7.54(\mathrm{~d}, 1 \mathrm{H}, J=1.9$, benzoxazolone- $H$ ), $7.80(\mathrm{~d}, 2 \mathrm{H}, J=8.8$, phenyl- $H$ ), 8.22 (d, 2H, $J=8.7$, phenylH), 8.37 (d, $2 \mathrm{H}, J=8.7$, phenyl- $H$, min), 10.98 (brs, $1 \mathrm{H}, \mathrm{NH})$ ppm. ESI-MS $m / z(\%): 348\left([\mathrm{M}+\mathrm{H}]^{+}, 38\right)$. Anal. calcd. for $\mathrm{C}_{15} \mathrm{H}_{10} \mathrm{~N}_{3} \mathrm{O}_{5} \mathrm{Cl}$ : C, 51.81; H, 2.90; N, 12.08. Found: C, 52.14; $\mathrm{H}, 3.01 ; \mathrm{N}, 12.40$.

3-(5-Chloro-2-oxobenzo[d]oxazol-3(2H)-yl)- $\mathrm{N}$ phenylpropanamide (5): Yield $83 \%$; reflux time: $42 \mathrm{~h}$; m.p. $209{ }^{\circ} \mathrm{C}$; IR (KBr, $v_{\max }, \mathrm{cm}^{-1}$ ) (FT/ATR): $3244,1771,1676 .{ }^{1} \mathrm{H}$ NMR (DMSO- $\left.d_{6}\right): \delta 2.78\left(\mathrm{t}, 2 \mathrm{H}, J=6.5, \mathrm{CH}_{2} \mathrm{CO}\right), 4.11(\mathrm{t}, 2 \mathrm{H}$, $\left.J=6.7, \mathrm{NCH}_{2}\right), 7.02(\mathrm{t}, 1 \mathrm{H}, J=7.3$, benzoxazolone- $H), 7.14$ (dd, $1 \mathrm{H}, J=1.9 ; 7.9$, benzoxazolone- $H$ ), 7.27 (t, 2H, $J=7.8$, phenyl- $H$ ), 7.34 (d, $1 \mathrm{H}, J=8.7$, benzoxazolone- $H$ ), 7.48-7.51 (m, 3H, phenyl- $H$ ), 10.01 ( brs, $1 \mathrm{H}, \mathrm{NH}$ ) ppm. ESI-MS $\mathrm{m} / z$ (\%): $317\left([\mathrm{M}+\mathrm{H}]^{+}, 28\right), 319\left([\mathrm{M}+\mathrm{H}+2]^{+}, 6\right)$. Anal. calcd. for $\mathrm{C}_{16} \mathrm{H}_{13} \mathrm{~N}_{2} \mathrm{O}_{3} \mathrm{Cl} \cdot 0.4 \mathrm{C}_{2} \mathrm{H}_{6} \mathrm{O}: \mathrm{C}, 60.15 ; \mathrm{H}, 4.59 ; \mathrm{N}, 8.35$. Found: C, 60.58; H, 4.88; N, 8.26.

3-(5-Chloro-2-oxobenzo[d]oxazol-3(2H)-yl)- $\mathrm{N}$-(4methoxyphenyl)propanamide (6): Yield $80 \%$; reflux time: 6 h; m.p. $172{ }^{\circ} \mathrm{C}$; IR (KBr, $\left.v_{\max }, \mathrm{cm}^{-1}\right)$ (FT/ATR): 3353, 1754 , 1675. ${ }^{1} \mathrm{H}$ NMR (DMSO- $\left.d_{6}\right): \delta 2.72\left(\mathrm{t}, 2 \mathrm{H}, J=6.8, \mathrm{CH}_{2} \mathrm{CO}\right.$ ), $3.67\left(\mathrm{~s}, 3 \mathrm{H}, \mathrm{OCH}_{3}\right), 4.08\left(\mathrm{t}, 2 \mathrm{H}, J=6.6, \mathrm{NCH}_{2}\right), 6.82(\mathrm{~d}, 2 \mathrm{H}$, $J=8.9$, phenyl- $H$ ), $7.12(\mathrm{dd}, 1 \mathrm{H}, J=2.1$; 8.3, benzoxazolone$\mathrm{H}), 7.32(\mathrm{~d}, 1 \mathrm{H}, J=8.5$, benzoxazolone- $H), 7.37(\mathrm{~d}, 2 \mathrm{H}, J=$ 8.9, phenyl- $H$ ), 7.44 (d, $1 \mathrm{H}, J=1.9$, benzoxazolone- $H$ ), 9.85 
(brs, 1H, NH) ppm. ESI-MS m/z (\%): $347\left([\mathrm{M}+\mathrm{H}]^{+}, 18\right), 349$ $\left([\mathrm{M}+\mathrm{H}+2]^{+}, 6\right)$. Anal. calcd. for $\mathrm{C}_{17} \mathrm{H}_{15} \mathrm{~N}_{2} \mathrm{O}_{4} \mathrm{Cl} \cdot \mathrm{H}_{2} \mathrm{O}: \mathrm{C}, 55.97$; H, 4.70; N, 7.68. Found: C, 55.75; H, 4.37; N, 7.63.

3-(5-Chloro-2-oxobenzo[d]oxazol-3(2H)-yl)-N-(4chlorophenyl)propanamide (7): Yield $26 \%$; reflux time: 48 h; m.p. $186{ }^{\circ} \mathrm{C}$; IR (KBr, $v_{\max }, \mathrm{cm}^{-1}$ ) (FT/ATR): 3342, 1746, 1681. ${ }^{1} \mathrm{H}$ NMR (DMSO- $\left.d_{6}\right): \delta 2.80\left(\mathrm{t}, 2 \mathrm{H}, J=6.6, \mathrm{CH}_{2} \mathrm{CO}\right)$, $4.12\left(\mathrm{t}, 2 \mathrm{H}, J=6.6, \mathrm{NCH}_{2}\right), 7.14(\mathrm{dd}, 1 \mathrm{H}, J=2 ; 8.3$, benzoxazolone- $H)$, 7.32-7.35 (m, $3 H$, benzoxazolone- $H$, phenyl- $H$ ), 7.47 (d, $1 \mathrm{H}, J=2$, benzoxazolone- $H$ ), 7.53 (d, $2 \mathrm{H}, J=9.2$, phenyl- $H$ ), 10.15 (brs, $1 \mathrm{H}, \mathrm{NH}$ ) ppm. ESI-MS m/ $z(\%): 351\left([\mathrm{M}+\mathrm{H}]^{+}, 1\right)$. Anal. calcd. for $\mathrm{C}_{16} \mathrm{H}_{12} \mathrm{~N}_{2} \mathrm{O}_{3} \mathrm{Cl}_{2} \cdot \mathrm{H}_{2} \mathrm{O}$ : C, 52; H, 3.79; N, 7.58. Found: C, 51.90; H, 3.36; N, 7.38.

3-(5-Chloro-2-oxobenzo[d]oxazol-3(2H)-yl)- $\mathrm{N}$-(4nitrophenyl)propanamide (8): Yield $38 \%$; reflux time: 42 h; m.p. $272{ }^{\circ} \mathrm{C}$; IR (KBr, $v_{\max }, \mathrm{cm}^{-1}$ ) (FT/ATR): 3345, 1758, 1692. ${ }^{1} \mathrm{H}$ NMR (DMSO- $\left.d_{6}\right): \delta 2.88\left(\mathrm{t}, 2 \mathrm{H}, J=6.8, \mathrm{CH}_{2} \mathrm{CO}\right)$, $4.13\left(\mathrm{t}, 2 \mathrm{H}, J=6.6, \mathrm{NCH}_{2}\right), 7.15(\mathrm{dd}, 1 \mathrm{H}, J=2.3 ; 8.6$, benzoxazolone- $H$ ), 7.34 (d, $1 \mathrm{H}, J=8.6$, benzoxazolone- $H$ ), 7.49 (d, $1 \mathrm{H}, J=2.3$, benzoxazolone-H), 7.76 (d, $2 \mathrm{H}, J=9.2$, phenyl- $H$ ), 8.2 (d, $2 \mathrm{H}, J=9.2$, phenyl- $H$ ), 10.62 (brs, $1 \mathrm{H}$, $\mathrm{NH})$ ppm. ESI-MS m/z (\%): $362\left([\mathrm{M}+\mathrm{H}]^{+}, 18\right), 364([\mathrm{M}+\mathrm{H}$ $\left.+2]^{+}, 6\right)$. Anal. calcd. for $\mathrm{C}_{16} \mathrm{H}_{12} \mathrm{~N}_{3} \mathrm{O}_{5} \mathrm{Cl} \cdot 2.2 \mathrm{H}_{2} \mathrm{O}: \mathrm{C}, 47.83 ; \mathrm{H}$, 4.09 ; N, 10.46. Found: C, 47.51; H, 4; N, 10.64.

4-(5-Chloro-2-oxobenzo[d]oxazol-3(2H)-yl)- $\mathrm{N}$-phenylbutanamide (9): Yield $33 \%$; reflux time: 70 h; m.p. $173{ }^{\circ} \mathrm{C}$; IR (KBr, $\left.v_{\max }, \mathrm{cm}^{-1}\right)$ (FT/ATR): 3362, 1784, 1682. ${ }^{1} \mathrm{H}$ NMR (DMSO- $d_{6}$ ): $\delta 2$ (quin, $2 \mathrm{H}, J=6.9, \mathrm{CH}_{2} \mathrm{CH}_{2} \mathrm{CH}_{2}$ ), $2.40(\mathrm{t}, 2 \mathrm{H}$, $\left.J=7.3, \mathrm{CH}_{2} \mathrm{CO}\right), 3.87$ (t, $\left.2 \mathrm{H}, J=6.7, \mathrm{NCH}_{2}\right), 7.01(\mathrm{t}, 1 \mathrm{H}, J=$ 7.2 , benzoxazolone- $H), 7.16(\mathrm{dd}, 1 \mathrm{H}, J=1.9$; 7.9, benzoxazolone- $H$ ), $7.26(\mathrm{t}, 2 \mathrm{H}, J=7.7$, phenyl- $H), 7.35(\mathrm{~d}, 1 \mathrm{H}, J=$ 8.3, benzoxazolone- $H$ ), 7.51-7.54 (m, 3H, phenyl- $H$ ), 9.85 (brs, 1H, NH) ppm. ESI-MS m/z (\%): $331\left([\mathrm{M}+\mathrm{H}]^{+}, 4\right), 333$ $\left([\mathrm{M}+\mathrm{H}+2]^{+}, 1\right)$. Anal. calcd. for $\mathrm{C}_{17} \mathrm{H}_{15} \mathrm{~N}_{2} \mathrm{O}_{3} \mathrm{Cl}: \mathrm{C}, 61.73 ; \mathrm{H}$, 4.57 ; N, 8.47. Found: C, 62.02; H, 4.56; N, 8.60.

4-(5-Chloro-2-oxobenzo[d]oxazol-3(2H)-yl)- $\mathrm{N}$-(4methoxyphenyl)butanamide (10): Yield $25 \%$; reflux time: 35 h; m.p. $148^{\circ} \mathrm{C}$; IR (KBr, $v_{\max }, \mathrm{cm}^{-1}$ ) (FT/ATR): 3225, 1772, 1647. ${ }^{1} \mathrm{H}$ NMR (DMSO- $d_{6}$ ): $\delta 1.99$ (quin, $2 \mathrm{H}, J=6.9$, $\mathrm{CH}_{2} \mathrm{CH}_{2} \mathrm{CH}_{2}$ ), 2.36 (t, $2 \mathrm{H}, J=7.6, \mathrm{CH}_{2} \mathrm{CO}$ ), 3.70 (s, $3 \mathrm{H}$, $\left.\mathrm{OCH}_{3}\right), 3.87$ (t, $\left.2 \mathrm{H}, \mathrm{NCH}_{2}\right), 6.84$ (d, 2H, $J=8.8$, phenyl- $H$ ), 7.16 (dt, $1 \mathrm{H}, J=1.9 ; 8.3$, benzoxazolone-H), 7.35 (dd, 1H, $J$ $=1.5 ; 8.3$, benzoxazolone- $H), 7.43(\mathrm{~d}, 2 \mathrm{H}, J=8.8$, phenyl$\mathrm{H}), 7.51$ (d, $1 \mathrm{H}, J=1.9$, benzoxazolone- $H$ ), 9.71 (brs, $1 \mathrm{H}$, $\mathrm{NH})$ ppm. ESI-MS $m / z(\%): 361\left([\mathrm{M}+\mathrm{H}]^{+}, 1\right)$. Anal. calcd. for $\mathrm{C}_{18} \mathrm{H}_{17} \mathrm{~N}_{2} \mathrm{O}_{4} \mathrm{Cl} \cdot 0.2 \mathrm{H}_{2} \mathrm{O}$ : C, 59.24; H, 4.77; N, 7.67. Found: C, 58.98; H, 4.55; N, 7.84.

4-(5-Chloro-2-oxobenzo[d]oxazol-3(2H)-yl)- $\mathrm{N}$-(4chlorophenyl)butanamide (11): Yield $16 \%$; reflux time: 18 h; m.p. $145^{\circ} \mathrm{C}$; IR (KBr, $v_{\max }, \mathrm{cm}^{-1}$ ) (FT/ATR): 3235, 1769, 1654. ${ }^{1} \mathrm{H}$ NMR (DMSO- $d_{6}$ ): $\delta 1.98$ (quin, $2 \mathrm{H}, J=7.2$, $\mathrm{CH}_{2} \mathrm{CH}_{2} \mathrm{CH}_{2}$ ), 2.38 (t, $\left.2 \mathrm{H}, J=7.4, \mathrm{CH}_{2} \mathrm{CO}\right), 3.85$ (t, $2 \mathrm{H}, J=$ $\left.7.2, \mathrm{NCH}_{2}\right), 7.13(\mathrm{dd}, 1 \mathrm{H}, J=2.2 ; 8.3$, benzoxazolone- $H$ ), 7.29-7.34 (m, $1 \mathrm{H}$, benzoxazolone- $H), 7.30(\mathrm{~d}, 2 \mathrm{H}, J=9.2$, phenyl- $H$ ), 7.48 (d, $1 \mathrm{H}, J=1.9$, benzoxazolone- $H$ ), 7.54 (d, $2 \mathrm{H}, J=8.7$, phenyl- $H$ ), 9.98 (brs, $1 \mathrm{H}, \mathrm{NH}$ ) ppm. ESI-MS $m / z$ (\%): $365([\mathrm{M}+\mathrm{H}], 30), 367\left([\mathrm{M}+\mathrm{H}+2]^{+}, 10\right)$. Anal. calcd. for $\mathrm{C}_{17} \mathrm{H}_{14} \mathrm{~N}_{2} \mathrm{O}_{3} \mathrm{Cl}_{2}$ : C, 55.91; H, 3.86; N, 7.67. Found: C, 56.01; $\mathrm{H}, 3.80$; N, 7.77.
2,2-Diphenyl-1-picrylhydrazyl (DPPH), ascorbic acid, 2,2'-azinobis-(3-ethylbenzothiazoline-6-sulfonic acid) (ABTS), Trolox were purchased from Sigma, potassium persulphate was purchased from Merck.

Moleculer devices VersaMax microplate reader and Shimadzu UV-1601 UV-visible spectrophotometer. Antioxidant activities of all the synthesized compounds were performed using by spectrophotometric ABTS and DPPH methods.

2,2-Diphenyl-2-picrylhydrazyl hydrate radical scavenging activity: The hydrogen atom or electron donation ability of the compounds were measured from the bleaching of the purple colored methanol solution of 2,2-diphenyl-picrylhydrazyl (DPPH) ${ }^{22}$. Ascorbic acid was used as an antioxidant standard. The measurements were carried out in triplicate. The $\%$ DPPH radical scavenging activity was calculated using the following formula: DPPH radical scavenging activity $=\left[\left(\mathrm{A}_{\mathrm{B}^{-}}\right.\right.$ $\left.\left.A_{A}\right) / A_{B}\right] \times 100 \%$, where $A_{B}$ is the absorbance of the blank and $\mathrm{A}_{\mathrm{A}}$ is the absorbance of the sample.

2,2-Azinobis(3-ethylbenzothiazoline-6-sulfonic acid) radical scavenging activity: Trolox equivalent antioxidant capacity (TEAC)of compounds were determined by an improved assay based on the decolorization of the radical monocation of [2,2'-azinobis-(3-ethylbenzothiazoline-6sulfonic acid)] $\left(\mathrm{ABTS}^{+\bullet}\right)^{23}$. Trolox measured as an antioxidant standard.

The standard curve of the Trolox was obtained using a Trolox standard solution at various concentrations (0.25-2.5 $\mathrm{mM})$. The absorbances of the samples were compared to that of the standard curve and the antioxidant properties were expressed as mM Trolox equivalent. All measurements were carried out in triplicate.

\section{RESULTS AND DISCUSSION}

In this study, eleven 5-chloro-2(3H)-benzoxazolone derivatives bearing acetanilide, propionanilide and butrylanilide moiety at the third position have been synthesized to evaulate antioxidant activity profile. The synthesis of the title compounds was realized in two steps. For this purpose, in the first step, $\omega$-chloroanilides were prepared according to the reported procedure $^{21}$. In the second step, those intermediates were reacted with 5-chloro-2(3H)-benzoxazolone to furnish title compounds. The synthetic pathway is given in Scheme-I. The structures of the compounds were confirmed by spectral (IR, ${ }^{1} \mathrm{H}$ NMR and ESI Mass) and elemental analyses. According to the literature survey, compound $\mathbf{1}$ is reported derivative ${ }^{24}$; compound $\mathbf{3}$ is listed substance in the literature with the CAS registry number 353256-18-5, but corresponding scientific reference data are not available for this compound. The other rest of compounds are novel compounds.

The purity levels of compounds were determined by elemental analyses $(\mathrm{C}, \mathrm{H}, \mathrm{N})$ and results were within $\pm 0.4 \%$ of the calculated values.

In the IR spectra, the presence of $\mathrm{C}=\mathrm{O}$ stretching bands were the confirmative signals for the constructed functional groups in the title compounds. For instance, $\mathrm{C}=\mathrm{O}$ stretching bands of anilide and 2(3H)-benzoxazolone groups were observed between 1698-1647 and 1784-1746 $\mathrm{cm}^{-1}$, respectively. On the other hand, N-H strecthing bands of the secondary amide structure of the title compounds were observed ${ }^{25,26}$ between 3362 and $3225 \mathrm{~cm}^{-1}$. 
<smiles>COC(=O)C(=O)Nc1ccc(P)cc1</smiles><smiles>[2H]c1ccc(NC(=O)CNC(=O)Nc2ccc([2H])cc2)cc1</smiles>

Scheme-I: Synthesis of the compounds 1-11

${ }^{1} \mathrm{H}$ NMR spectra of the title compounds were consistent with expected resonance signals in term of chemical shifts and integrations. In addition, some expected minor signals were observed in spectra because of the rotamer formations which is well known and well described in the literature ${ }^{17}$.

The structure of the title compounds was further verified by ESI-mass spectra where the $\mathrm{m} / \mathrm{z}$ values of molecular ion peaks were in complete agreement with the calculated molecular weight for individual compounds.

Screening of antioxidant activity: The antioxidant activity profile of the synthesized compounds was determined by DPPH and ABTS free radical scavenging assays using ascorbic acid and trolox as reference compounds, respectively. Preliminary antioxidant activity screening results are presented in Tables 1 and 2.

According to the DPPH radical scavenging assay, butrylanilide derivatives (compounds 9-11) displayed activity with variable potency in all tested concentrations. While all members of propionylanilides (compounds 5-8) and acetanilides (compounds 1-4) were active in $1000 \mathrm{mg} / \mathrm{mL}$, their potency was gradually diminished by the decreasing concentration. For instance, only one acetanilide derivative (compound 2 ) displayed activity at $400 \mathrm{mg} / \mathrm{mL}$ and only one member of propionylanilides (compound 6) had activity at $50 \mathrm{mg} / \mathrm{mL}$. Interestingly, in all series and in all studied doses, the most active compound was compound 6 which belongs to the propionylanilide series.

The ABTS free radical scavenging activity of the synthesized compounds were determined and expressed as Trolox equivalent antioxidant capacity. According to the test result, all the compounds tested except for the compounds 3-5, 7 displayed antioxidant activity with variable potency and the test results were in complete agreement with the results of the DPPH radical scavenging assay. The most active compound in the series was compound $\mathbf{6}$ which was also the corresponding leading compound in DPPH radical scavenging assay.

The antioxidant activity results of the designed 5-chloro2(3H)-benzoxazolone derivatives in both tests were well correlated and provided parallel data. Generally, in the light of the screening results, it can be speculated that the number of carbon atoms of the studied benzoxazolone-3-alkananilide homolog series seems critical for determining the potency of the compounds. Consequently, the screening data pointed out compound $\mathbf{6}$ as the most active compound in both tests.

\begin{tabular}{cccccc}
\hline \multicolumn{6}{c}{ TABLE-1 } \\
\multicolumn{5}{c}{ DPPH RADICAL SCAVENGING } \\
\hline \multicolumn{6}{c}{ ACTIVITY (\%) OF TITLE COMPOUNDS } \\
\hline Compds. & $50(\mu \mathrm{g} / \mathrm{mL})$ & 100 & 200 & 400 & 1000 \\
& & $(\mu \mathrm{g} / \mathrm{mL})$ & $(\mu \mathrm{g} / \mathrm{mL})$ & $(\mu \mathrm{g} / \mathrm{mL})$ & $(\mu \mathrm{g} / \mathrm{mL})$ \\
\hline $\mathbf{1}$ & - & - & - & - & $18.74 \pm 0.69$ \\
$\mathbf{2}$ & - & - & - & $6.60 \pm 0.71$ & $4.34 \pm 0.62$ \\
$\mathbf{3}$ & - & - & - & - & $1.70 \pm 0.70$ \\
$\mathbf{4}$ & - & - & - & - & $0.69 \pm 0.20$ \\
$\mathbf{5}$ & - & - & - & - & $4.07 \pm 1.67$ \\
$\mathbf{6}$ & $9.16 \pm 0.32$ & $14.25 \pm 1.2$ & $24.23 \pm 0.63$ & $51.15 \pm 0.54$ & $60.32 \pm 0.87$ \\
$\mathbf{7}$ & - & - & - & - & $5.53 \pm 0.91$ \\
$\mathbf{8}$ & - & - & - & - & $5.05 \pm 0.78$ \\
$\mathbf{9}$ & $1.42 \pm 0.26$ & $2.87 \pm 0.12$ & $4.94 \pm 0.51$ & $13.14 \pm 0.4$ & $14.98 \pm 1.28$ \\
$\mathbf{1 0}$ & $3.20 \pm 0.82$ & $1.65 \pm 0.03$ & $1.19 \pm 0.17$ & $6.85 \pm 1.6$ & $12.42 \pm 0.8$ \\
$\mathbf{1 1}$ & $4.90 \pm 0.9$ & $6.72 \pm 0.38$ & $15.09 \pm 1.7$ & $25.69 \pm 0.38$ & $45.64 \pm 1.59$ \\
Ascorbic & $89.40 \pm 0.39$ & $94.60 \pm 0.47$ & $99.30 \pm 0.4$ & $99.50 \pm 0.32$ & $99.50 \pm 0.32$ \\
acid & & & & \\
\hline
\end{tabular}

(-) Showed no scavenging activity. The values were the means of three replicates \pm S.D.

\begin{tabular}{|c|c|}
\hline \multicolumn{2}{|c|}{$\begin{array}{c}\text { TABLE-2 } \\
\text { TROLOX EQUIVALENT ANTIOXIDANT } \\
\text { CAPACITY (TEAC) VALUES OF TITLE COMPOUNDS }\end{array}$} \\
\hline Compound & ${ }^{*}$ TEAC (mM) \\
\hline 1 & $0.28 \pm 0.006$ \\
\hline 2 & $0.20 \pm 0.004$ \\
\hline 3 & - \\
\hline 4 & - \\
\hline 5 & - \\
\hline 6 & $0.75 \pm 0.004$ \\
\hline 7 & - \\
\hline 8 & $0.22 \pm 0.009$ \\
\hline 9 & $0.26 \pm 0.013$ \\
\hline 10 & $0.29 \pm 0.014$ \\
\hline 11 & $0.56 \pm 0.010$ \\
\hline
\end{tabular}

TEAC is the $1 \mathrm{mM}$ concentration of a Trolox solution having the antioxidant capacity equivalent to $1 \mathrm{mg} / \mathrm{mL}$ solution of the compounds

\section{ACKNOWLEDGEMENTS}

This study was supported by research grants from Ege University (Project Number: 10/Ecz/017). The authors thank the Pharmaceutical Sciences Research Centre (FABAL) at Ege University Faculty of Pharmacy for spectral analyses of the compounds.

\section{REFERENCES}

1. M. Valko, C.J. Rhodes, J. Moncol, M. Izakovic and M. Mazur, Chem. Biol. Interact., 160, 1 (2006).

2. W. Droge, Physiol. Rev., 82, 47 (2002).

3. D.V. Ratnam, D.D. Ankola, V. Bhardwaj, D.K. Sahana and M.N.V.R. Kumar, J. Control. Release, 113, 189 (2006).

4. R. Hardeland, Endocrine, 27(2), 119, (2005).

5. E.R.V. Rios, E.T. Venancio, N.F.M. Rocha, D.J. Woods, S. Vasconcelos, D. Macedo, F.C.F. Sousa and M.M.F. Fonteles, Int. J. Neurosci., 120, 583 (2010).

6. R. Hardeland, S.R. Pandi-Perumal and D.P. Cardinali, Int. J. Biochem. Cell Biol., 38, 313 (2006).

7. L.A. Thompson and A.J. Ellman, Chem. Rev., 96, 555 (1996).

8. I. Chiarotto, M. Feroci, M. Orsini, G. Sotgiu and A. Inesi, Tetrahedron, 65, 3704 (2009).

9. B.-L. Deng, M.D. Cullen, Z. Zhou, T.L. Hartman, R.W. Buckheit Jr., C. Pannecouque, E.D. Clercq, P.E. Fanwick and M. Cushman, Bioorg. Med. Chem., 14, 2366 (2006). 
10. Y. Ivanova, G. Momekov, O. Petrov, M. Karaivanova and V. Kalcheva, Eur. J. Med. Chem., 42, 1382 (2007).

11. S. Unlu, T. Onkol, Y. Dundar, B. Okcelik, E. Kupeli, E. Yesilada, N. Noyanalpan and M.F. Sahin, Arch. Pharm., 336, 353 (2003).

12. M. Koksal, N. Gokhan, E. Kupeli, E. Yesilada and H. Erdogan, Arch. Pharm., 338, 117 (2005)

13. T. Onkol, S. Ito, E. Yildirim, K. Erol and M.F. Sahin, Arch. Pharm., 334, 17 (2001).

14. M. Koksal, N. Gokhan, H. Erdogan, M. Ozalp and M. Ekizoglu, Il Farmaco, 57, 535 (2002)

15. H. Ucar, K. Van derpoorten, S. Cacciaguerra, S. Spampinato, J.P. Stables, P. Depovere, M. Isa, B. Masereel, J. Delarge and J.H. Poupaert, J. Med. Chem., 41, 1138 (1998).

16. M. Courtois, Z. Mincheva, F. Andreu, M. Rideau and M.C. ViaudMassuard, J. Enzyme Inhib. Med. Chem., 19, 559 (2004).

17. Z. Soyer, M. Bas, A. Pabuçcuoglu and V. Pabuçcuoglu, Arch. Pharm., 338, 405 (2005).
18. T.B. Ng, F. Liu and L. Zhao, J. Neural Transm., 107, 1243 (2000).

19. H. Orhan, D.S. Dogruer, B. Çakir, G. Sahin and M.F. Sahin, Exp. Toxicol. Pathol., 51, 397 (1999).

20. R.J. Nachman, J. Heterocycl. Chem., 19, 1545 (1982).

21. D.E.F. Gangi, J. Am. Pharm. Assoc., XLIV, 135 (1955).

22. K.J. Wang, Y.J. Zhang and C.R. Yang, Chem. Biodivers., 3, 1317 (2006).

23. R. Re, N. Pellegrini, A. Proteggente, A. Pannala, M. Yang and C. RiceEvans, Free Radic. Biol. Med., 26, 1231 (1999).

24. U. Calis, H.G. Pilli and F. Ozkanli, Hacettepe Univ. J. Fac. Pharmacy, 12, 71 (1992).

25. M. Hesse, H. Meier and B. Zeeh, Spectroscopic Methods in Organic Chemistry, Georg Thieme Verlag: Stutgart, New York (1997).

26. K. Nakanishi and P.H. Solomon, Infrared Absorption Spectroscopy, Holden-Day Inc. San Francisco, edn. 2 (1977). 